\title{
Las primeras declaraciones de los profesionales de la salud del Hospital de Campo de Mayo. Una aproximación a las actitudes sociales en la última Dictadura
}

\section{The first depositions of the Hospital de Campo de Mayo's health professionals. An overview to the social attitudes during the last military dictatoship in Argentina}

\author{
Clara Sarsale \\ Instituto de Desarrollo Económico y Social \\ Universidad Nacional General Sarmiento / Universidad de Buenos Aires, Argentina \\ csarsale@gmail.com
}

\begin{abstract}
Resumen
En los primeros meses del gobierno de Raúl Alfonsín, en 1984, la Comisión Nacional sobre la Desaparición de Personas comenzó a recibir testimonios de todo aquel que tuviera información sobre lo acontecido en los años de dictadura. Entre los declarantes se encontraron familiares de desaparecidos, sobrevivientes, entre otros testigos menos usuales. Allí residirá el foco de este trabajo: en el testimonio de algunos de los profesionales de la salud del Hospital Militar de Campo de Mayo frente a la CONADEP. Estos profesionales, asistieron los partos de mujeres que se encontraban secuestradas por la dictadura. Como se verá en el artículo, ubicados en el corazón del sistema represivo, estos profesionales tuvieron diversas respuestas frente a lo que estaba ocurriendo. Su estudio, permite abrir nuevas preguntas sobre las actitudes sociales en dictadura.
\end{abstract}

Palabras clave

Dictadura; Actitudes Sociales; Testimonios; Profesionales de la salud; Campo de Mayo

\begin{abstract}
On the first months of Raúl Alfonsín government, in 1984, the Comisión Nacional sobre la Desaparición de Personas (CONADEP) started gathering testimonies from anyone that had information about what happened during the military dictatorship. The deponents were relatives ofdesaparecidos, survivors, among other unusual witnesses. That is the focus of this work; the testimonies given to the CONADEP by some of the health professionals of the Hospital Militar Campo de Mayo. These professionals assisted the births of women that were kidnapped by the dictatorship. As we will show on this paper, acting on the heart of the repressive system, they had different responses towards what happened which permits to ask new questions regarding the social attitudes during these years.
\end{abstract}

\section{Keywords}

Social attitudes; Testimonies; Health professionals; Campo de Mayo

Esta obra está sujeta a la Licencia Reconocimiento-NoComercial-CompartirIgual 4.0 Internacional de Creative Commons. http://creativecommons.org/licenses/by-nc-sa/4.0/ (cc) EYY-NC-SA 


\section{Clara Sarsale}

\section{Introducción}

En este texto se recorrerá brevemente algunos debates historiográficos que han problematizado la cuestión del consenso, principalmente en el nazismo. Luego, a la luz de esas reflexiones, analizaré los testimonios de profesionales de la salud que trabajaron en el Hospital Militar de Campo de Mayo durante la dictadura. Estos testimonios, posibilitarán la reflexión sobre la diversidad de actitudes sociales que .tuvieron lugar en la sociedad argentina durante la última dictadura militar.

¿Qué lugar ocupa el consenso en la instauración y sostenimiento de una dictadura? ¿En qué medida cada régimen se propone generar ese apoyo? ¿Qué lugar y qué posibilidades de visibilización tienen, por el contrario, la oposición y la resistencia? Estas preguntas básicas han sido formuladas tarde o temprano en todos los países que han atravesado procesos dictatoriales. Sin dudas, el contexto histórico local ${ }^{1}$ es central para comprender cuándo surgen esas preguntas en cada una de esas sociedades. Sin embargo, se puede afirmar que esas preguntas encuentran condiciones de posibilidad en la medida en que se ha llegado a algún grado de acuerdo respecto de quiénes fueron los principales responsables. En el caso alemán, por ejemplo, las investigaciones sobre vida cotidiana comienzan recién a fines de los setentas ${ }^{2}$. La Alltagsgeschichte o la historia de la vida cotidiana conlleva un supuesto: "por debajo de la barbarie y del horror del régimen había patrones de normalidad social que fueron, por supuesto afectados por el nazismo (...) pero que eran anteriores a él y lo sobrevivieron" (Kershaw, XX, 291) ${ }^{3}$. En este sentido, las preguntas sobre la vida cotidiana y las actitudes sociales implican la inscripción de un acontecimiento histórico específico (el nazismo, por ejemplo) en el devenir histórico de Alemania. El otro supuesto que subyace es la consideración de los individuos como sujetos que toman decisiones y actúan, en parte, de manera racional. En palabras de Lüdtke, referente de la Alltagsgeschichte, ésta "se centra en la práctica de la multitud. (...) La práctica hace alusión a la forma en que los hombres se apropian de las condiciones en las que viven, producen experiencias, utilizan modos de expresión e interpretaciones - y las actúan nuevamente por su parte-. En el proceso de apropiación los agentes se convierten en actores que

\footnotetext{
1 Daniel Lvovich (2008) señala como un punto importante a tener en cuenta para analizar el desarrollo de estudios sobre las actitudes sociales, el lugar que ocuparon en el relato post dictatorial las organizaciones de resistencia. Para el caso español, por ejemplo, la salida pactada del franquismo conllevó primordialmente una serie de políticas de olvido que anularon la apertura de investigaciones. En el caso italiano, por el contrario, la memoria de los resistentes tuvo un lugar central en la narrativa posterior lo que habilitó a una discusión más temprana sobre lo sucedido.

${ }^{2}$ Es interesante señalar que el desarrollo de esta escuela historiográfica coincide con un momento de expansión del debate público sobre lo ocurrido en la Alemania nazi. En este sentido, según plantea Baer cumple un rol de importancia la transmisión de la serie Holocausto (1979) y el cambio generacional que posibilita la realización de preguntas críticas por parte de los hijos $(2006,119)$.

${ }^{3}$ Frente al planteo de la Alltagsgeschichte, Friëdlander, señala el riesgo de que: "en una perspectiva general del Tercer Reich apoyada en la normalización y la relativización de la era nazi, (...) la tendencia a cargar el extremo de la "normalidad" del continuo difícilmente podía ser evitada." (Kershaw, 2004, 294).
} 


\section{Las primeras declaraciones de los profesionales de la salud del Hospital de Campo de Mayo}

interpretan y se muestran, presionan o rechazan" (Lüdtke, 1995,49). Desde esta perspectiva, entonces, las actitudes sociales no aparecen como reflejo del poder hegemónico sino que son actos de apropiación y resignificación del mismo. Teniendo en cuenta esto, las preguntas que propone la Alltagsgeschichte son, en última instancia, preguntas por las condiciones de realización del poder: "de qué modo se imponía este dominio desde arriba y se (co)producía al mismo tiempo desde abajo" (Lüdtke, 1995,60).

Otro hito en la producción historiográfica sobre el nazismo, fue Aquellos hombres grises. El batallón 101 y la Solución Final en Polonia (1992) de Christopher Browning. El autor, a partir de un minucioso análisis de fuentes, da cuenta de las diversas posibilidades que tuvieron los integrantes del Batallón 101 para decidir participar o no en el asesinato de judíos. A partir del análisis de las fuentes, Browning establece que sólo una minoría eligió apartarse de la tarea encomendada. Además, plantea que aún quienes si participaron de la matanza tuvieron que consumir grandes cantidades de alcohol para atravesar la situación o bien que, una vez comenzada la tarea, trataron de evadirse de la misma. Lo que pone de relieve Browning con su célebre estudio, es la inexistencia de una linealidad necesaria en el accionar de los ejecutores. A partir de su análisis, puede afirmarse la importancia de analizar un caso específico como prisma para mirar los màrgenes de acción en una dictadura y complejizar su estudio utilizando una mayor cantidad de variables para explicar el comportamiento que no se limiten a opciones dicotómicas como víctima o victimario.

En 1996 se dio inicio a un fuerte debate en el campo historiográfico con la publicación del libro de Daniel Goldhagen "Los verdugos voluntarios de Hitler". El libro, que resultó un absoluto éxito editorial entre el público no especializado, partía de las mismas fuentes que Browning pero alcanzaba conclusiones totalmente diferentes. Como señala Finchelstein, "Goldhagen postula la existencia de un continuum antisemita específicamente alemán, un super pogrom que comienza en el medioevo y desemboca en un "antisemitismo esencialista" que hace que cualquier alemán quiera matar judíos” (Finchelstein, 1999,37). Más allá de las críticas metodológicas a la investigación de Goldhagen, lo que está en el centro de la discusión es la posibilidad de acción (de resistencia, aceptación, resignificación) por parte de los sujetos en un proceso dictatorial. Desde la mirada esencialista que propone Goldhagen los sujetos resultan meros reproductores de una cultura (la antisemita en el caso del nazismo) que han heredado y frente a la que son necesariamente pasivos.

Para el análisis que propongo en este trabajo, retomo la líneas de interpretación planteadas por la Alltagsgeschichte y la investigación de C. Browning, en la que los individuos son sujetos activos y por lo tanto, es necesario pensar con qué categorías puede abordarse sus acciones en contextos dictatoriales y en qué medida resultan aplicables en cada caso histórico. El historiador español Saz Campos, plantea una pregunta dilemática: el consenso, ¿resulta un concepto iluminador o, por el contrario "relega a segundo plano el factor represivo, mucho 


\section{Clara Sarsale}

más importante en la sustentación de la dictadura (...) [y] conduc[e] a una minusvaloración de un cúmulo de actitudes de resentimiento y rechazo"?(2004, 171) Saz Campos propone al consenso como una categoría para estudiar cómo construye cada dictadura su legitimidad frente a la sociedad al tiempo que señala que "el ciudadano experimenta actitudes que no se pueden reducir a la noción de consenso porque puede expresar elementos de rechazo, elementos de cierta resistencia, que luego pueden conducir a una identificación con el régimen o a una oposición abierta" $(157,2014)$. Teniendo en cuenta esto es necesario, como señala Hernández Burgos "no sólo atender a la recepción de los discursos emitidos "desde arriba", sino comprender que estos también son coproducidos "desde abajo" (...)" $(2012,27)$.

Tanto los planteos de la Alltagsgeschichte como los de Saz Campos y Hernández Burgos, invitan a pensar en la necesidad de realizar estudios monográficos para poder dar cuenta de la diversidad de actitudes sociales en regímenes dictatoriales.

\section{Las fuentes en los estudios sobre actitudes sociales.}

Como se ha plasmado en el apartado anterior, la posibilidad de asir las actitudes sociales, ver la producción de acciones y sentidos y la apropiación y transformación de éstas por distintos sujetos de la sociedad es sumamente compleja. Desde luego, las fuentes orales deberán ocupar un lugar preponderante en este tipo de estudios dado que permiten acceder a un relato experiencial en primera persona, y enfatizar determinados aspectos relevantes para la investigación en proceso. Sin embargo, en este trabajo, dada la intención de enfocar en un momento particular de la historia argentina y de analizar las variaciones en los testimonios según el contexto de enunciación, se optó por trabajar sólo con testimonios judiciales. Esta decisión no ignora que la escena judicial está sumamente reglada y marcada por un funcionamiento jerárquico que impacta en el los testimoniantes. Las reglas que organizan la escena judicial, descoloca e incomoda a quienes no las manejan, pudiendo - por ejemplo - reducir la fluidez en el habla de quien declara (Kaufman, 1991; Sarrabayrouse, 2004); además en los tribunales está siempre la posibilidad de autoincriminarse en un delito por lo que los testimoniantes desarrollan diversas estrategias a la hora de expresarse. Por último, y tal como se pondrá de relieve en el próximo apartado, el contexto de producción de los testimonios tiene un rol importante en el contenido del mismo. Todos estos señalamientos son un llamado de atención metodológico que tratará de contemplarse en el uso de las fuentes que se hace en el presente artículo.

En este trabajo, el análisis de estas fuentes permitirá ver ciertos matices respecto a la interpretación dominante en la década del 80 sobre el rol de la 


\section{Las primeras declaraciones de los profesionales de la salud del Hospital de Campo de Mayo}

sociedad; en el relato sobre el pasado reciente que se plasma en el Nunca más (1984), "la "sociedad" es retratada como conjunto en una posición dual: como posible víctima y como observadora ajena que, si justifica el horror, es debido al terror imperante. En ambos casos, inocente del ejercicio de la violencia y del horror" (Crenzel, 2007,52). Ahora bien, en las declaraciones en las que se basa el Nunca más y las que se producen en los meses siguientes a su publicación, se pueden rastrear - como se verá - una diversidad de actitudes sociales. Aquí radica entonces, el interés por el uso de este tipo de fuentes en este trabajo.

\section{Campo de Mayo: los primeros testimonios sobre la maternidad clandestina.}

En 1901, bajo la presidencia Julio Argentino Roca, se aprobó la compra de los terrenos que hoy conforman Campo de Mayo para la construcción de una enorme guarnición militar. Tres años más tarde, en 1904, se aprobó la construcción del Hospital Militar de Campo de Mayo con asistencia básica. Con el paso de los años, se incorporaron al Hospital nuevas especialidades como traumatología y dermatología entre otras. Según se destaca en su web, el Hospital brindó servicios a la comunidad luego del terremoto en San Juan (1944), en el brote de poliomelitis de 1956, en el conflicto fronterizo con Chile y tras la Guerra de Malvinas. Aunque en esta breve historización no aparece mencionada, desde el regreso de la democracia se conoce que allí funcionó una maternidad clandestina durante la dictadura militar que comenzó en $1977^{5}$.

A partir de la vuelta de la democracia y la conformación de la CONADEP comenzaron las denuncias sobre la maternidad clandestina de Campo de Mayo. Un grupo de enfermeras ${ }^{6}$ y una médica concurrieron tempranamente a declarar a la CONADEP$^{7}$ (1984). Luego de esos primeros testimonios, otros profesionales continuaron declarando tanto la justicia civil y la justicia militar. Según la información recabada hasta el momento y teniendo en cuenta los juicios que tratan la apropiación de niños en general y no apropiaciones particulares, los

\footnotetext{
4 El informe Nunca más publicado por EUDEBA en 1984 fue el producto de la investigación realizada por la Comisión sobre la desaparición de personas (CONADEP de ahora en adelante) que conformó el Poder Ejecutivo para averiguar sobre el destino de los desaparecidos.

${ }^{5}$ Anteriormente hubo partos en el Centro Clandestino que funcionaba en Campo de Mayo. En el Hospital, según se sabe los partos comenzaron en 1977.

${ }^{6}$ El primer grupo estuvo compuesto por voluntarios, y luego, un segundo grupo compuesto por otros miembros del Hospital fueron convocados a declarar por la Comisión Nacional sobre la desaparición de Personas (CONADEP) creada en 1983 para investigar lo acontecido con las personas desaparecidas.

${ }^{7}$ Sus testimonios fueron posteriormente publicados en el Capítulo 2 del "Nunca más" dedicado a las víctimas en un apartado denominado "Niños desaparecidos y embarazadas" en el subapartado "El Hospital Campo de Mayo".
} 


\section{Clara Sarsale}

profesionales de Campo de Mayo han declarado frente al Poder Judicial de la Nación en 1984, a la Justicia Militar ${ }^{8}$ (1985) y luego en 1998, 2011 y $2014^{9}$.

En cada uno de los juicios mencionados, aunque lo que se investiga y juzga es el funcionamiento y los nacimientos en la maternidad clandestina del Hospital de Campo de Mayo, se abre un resquicio, a partir de las declaraciones, para analizar las actitudes sociales adoptadas por los profesionales que trabajaron allí. Teniendo en cuenta esto, es importante destacar que tanto las enfermeras como los médicos del Hospital de Campo de Mayo, actuaron en tanto profesionales de la salud; esto es, sus superiores los requirieron "no (...) una ejecución mecánica, sino la utilización de su experiencia y de su saber especializado. La actividad y la disposición a involucrarse, el desarrollo creativo de las instrucciones eran y estaban consideradas como imprescindibles" (Lüdtke 1995, 64). Esta afirmación no desconoce el contexto represivo que se vivía, si no que pretende poner en foco la agencia de los sujetos en ese contexto.

Teniendo en cuenta esta perspectiva, se analizarán algunos de los primeros testimonios brindados por profesionales de la salud (una obstétrica, una médica tocoginecóloga y un enfermero radiólogo) en 1984 y 1985.

Estas primeras declaraciones tuvieron un peso fundamental en la construcción de la historia de la maternidad clandestina de Campo de Mayo ya que - junto con otros testimonios - sentaron las bases sobre las cuales se investigó y que permitieron posteriormente condenar a algunos de los responsables civiles y militares en los juicios de 2011 y $2014^{10}$.

¿Qué información aparece en esas primeras declaraciones? Los primeros testimonios, en primer lugar, fueron la comprobación de la existencia de la maternidad clandestina de Campo de Mayo. Asimismo, permitieron saber que 1977 y 1978 fueron los años de más actividad en la maternidad clandestina. En segundo lugar, posibilitaron conocer, al menos en parte, el dispositivo de

\footnotetext{
${ }^{8}$ Se trata de declaraciones de carácter testimonial tomadas en Campo de Mayo, de acuerdo a lo regulado por el Código Penal Argentino y del Código de Justicia Militar donde se los interroga sobre los dichos frente a la CONADEP.

${ }^{9}$ En el marco de las leyes de obediencia debida y punto final y el imperio de los indultos, una de las estrategias judiciales de los organismos de DD.HH. fue querellar por la apropiación de menores durante la dictadura militar. En 1998, el juez Marquevich encarcela a Videla por el delito de apropiación. Ese juicio, será la base documental sobre el que se estructurará posteriormente el juicio denominado "Plan sistemático de apropiación de bebés" llevado a cabo por el TOF 6 en el 2011. Finalmente, en 2014 se realiza el juicio donde además de las jerarquías militares se juzga a médicos y una enfermera de Campo de Mayo.

${ }^{10}$ El juicio de 2011 tramitado por el TOF 6 de la Ciudad de Buenos Aires, fue conocido como "Plan sistemático de apropiación de menores" donde se juzgó a Jorge Rafael Videla, Reynaldo Benito Bignone, Santiago Omar Riveros, Antonio Vañek, Jorge Eduardo Acosta, Rubén Oscar Franco, Juan Antonio Azic, Jorge Luis Magnacco, Inés Susana Colombo, Víctor Alejandro Gallo, Eduardo Alfredo Ruffo. El juicio de 2014, tramitado por el mismo juzgado, fue conocido como "Juicio a los médicos de Campo de Mayo" y se juzgó a Santiago Omar Riveros, Reynaldo Benito Bignone, Raúl Eugenio Martín, Norberto Atilio Bianco y Luisa Yolanda Arroche. Además de los juicios mencionados en este texto, en 2014 fue condenada Lidia Fanni Villavicencio, obstetra del Hospital de Campo de Mayo, por firmar el certificado falso de nacimiento de Catalina de Sanctis Ovando
} 


\section{Las primeras declaraciones de los profesionales de la salud del Hospital de Campo de Mayo}

funcionamiento de la maternidad. El pabellón de epidemiología fue el lugar donde fueron recluidas las embarazadas (según algunos relatos también se alojaron allí heridos). Las mujeres embarazadas eran llevadas allí desde "El Campito", uno de los centros clandestinos que funcionaban en Campo de Mayo. Una vez en epidemiología, las mujeres eran retenidas en habitaciones separadas con baño y ventanas tapiadas; en algunas descripciones se menciona también que estaban con los ojos vendados. Allí, los profesionales del Hospital llevaban el control de la salud de las mujeres y el avance del embarazo, atendían los partos y hacían las curaciones postparto. En los casos en que era necesario practicar una cesárea, las mujeres eran trasladadas al edificio central en donde funcionaba el servicio de maternidad del Hospital en el que también eran atendidas las esposas de los militares.

Las detenidas y sus bebés eran identificadas como N.N., y las indicaciones respecto de la medicación anotada en una hoja en blanco ${ }^{11}$ sin dejar - en la mayor parte de los casos- registro en el libro de parto ni historia clínica. El pabellón tenía custodia militar en la puerta de forma constante. Los profesionales civiles debían entrar siempre al sector de epidemiología con personal militar; por su parte, los médicos militares se manejaban con libertad pudiendo entrar y salir en cualquier momento.

Las primeras declaraciones fueron hechas ante la CONADEP y la información que brindaron tuvo un grado mucho mayor de generalidad - esto puede verse por ejemplo, en la extensión de las mismos no supera las dos páginas que las que les demandó la Justicia Militar donde se interrogó con minuciosidad sobre algunos aspectos como el trato médico dado a las embarazadas. No es esta la única diferencia entre las declaraciones: si bien la Justicia Militar toma como base lo testimoniado en CONADEP, en el interrogatorio militar puede advertirse un halo persecutorio, intimidador. En este sentido, a la hora de analizar los testimonios producidos en la instancia judicial debe advertirse el contexto en el cual éstos han sido producidos. Luego de siete años en el gobierno, el poder político y social que conservaban las FF.AA. y su capacidad de amedrentar era significativa. Además, algunos de los testimoniantes todavía trabajaban en el Hospital de Campo de Mayo por lo que el Ejército seguía siendo su empleador.

En este trabajo, tomaré tres testimonios de distintos profesionales del Hospital: Jorge Luis Eposto, la Dra. Bonsignore de Petrillo y la obstétrica Valaris para reflexionar sobre las diferentes actitudes sociales adoptadas. Si bien no pretende ser una muestra concluyente se eligieron estos tres testimonios por

\footnotetext{
11 Esta situación tiene algunas excepciones; el parto de Silvia Mónica Quintela Dallasta fue anotado fuera de fecha en el libro de partos fraguando su nombre y poniendo en su lugar el de Susana Colombo, la apropiadora de Francisco Madariaga Quintela. El nacimiento de Catalina de Sanctis Ovando también fue anotado, atribuyéndole la maternidad a su apropiadora, Francisca Miorilla. Además, el parto de Myriam Ovando aparece registrado pero según el libro de partos, se trató de un legrado de 45 días.
} 


\section{Clara Sarsale}

tratarse de distintas especialidades y además por las diferencias de género y la pertenencia o no a la corporación militar.

Jorge Luis Eposto fue técnico radiólogo y enfermero con grado de cabo primero en el Hospital Militar; como tantos otros, Eposto vivía en el barrio de Sub oficiales de Campo de Mayo. Sin embargo, es el único militar que el 30 de enero de 1984 se acercó a prestar testimonio en la CONADEP. Lo hizo, según consigna el acta de su declaración, de forma espontánea y no, como harán luego otros militares, por la convocatoria a declarar que extiende CONADEP. Al igual que las otras dos testimoniantes que se analizarán en este trabajo, Eposto señaló al pabellón de epidemiología como el lugar donde se mantenía a las embarazadas internadas y atadas. Sin embargo, en sus palabras se filtran las huellas de la época: a las mujeres embarazadas las define como extremistas.

Si bien él vió cuatro o cinco mujeres, sabe por sus compañeros que hubo más embarazadas en epidemiología. También declaró que éstas eran trasladadas al Hospital al momento del parto y que las madres eran separadas de sus bebés luego del alumbramiento.

Por su pertenencia a la corporación militar, Eposto accedía a otro tipo de información y a otros informantes. Por ejemplo, vivir en el barrio de sub oficiales le permitió dar cuenta de la existencia de lo que luego se identificaría como "vuelos de la muerte". Según su relato, el veía cada vez que estaba de guardia y oía desde su casa, un avión dirigiéndose siempre en la misma dirección que pasaba alrededor de las 23:30 y regresaba a la una de la madrugada. Según se decía entre el personal del Hospital, en los aviones trasladaban personas para tirarlas al mar. En su declaración, Eposto también mencionó la existencia de Grupos de tareas. Según narra, se enteró de esto por un colega enfermero radiólogo que participaba de las acciones del grupo de tareas colaborando con su conocimiento profesional en caso de ser necesario. Como puede notarse, el testimonio de este enfermero se fue conformando entrelazando experiencias personales con información o rumores circulantes.

Las declaraciones hechas ante CONADEP por Eposto fueron ratificadas en instancia judicial en agosto del 1984. Allí agrega a su declaración una denuncia por haber recibido una amenaza personal de muerte. Si bien no se dispone de más información que los dichos de Eposto, resulta una información significativa respecto del contexto en el que se realizaban las declaraciones.

Como era de prever, las declaraciones de Eposto frente a la Justicia militar tienen un carácter bastante diferente dado que es la propia corporación militar la que está siendo denunciada. Esto hace que quienes están llevando adelante el interrogatorio sean, simultáneamente, juez y parte. La búsqueda de información precisa en el interrogatorio, es enfática. En el contexto militar, la clandestinidad como parte del dispositivo represivo parece no ser tenido en cuenta a la hora de preguntar. El testimonio de Eposto resulta entonces mucho más endeble, plagado de referencias como "por haber escuchado un comentario proveniente de una persona o personas que en este momento no puedo recordar" o "dicha versión 


\section{Las primeras declaraciones de los profesionales de la salud del Hospital de Campo de Mayo}

llegó a través de algún comentario escuchado dentro del Hospital" (Eposto, JM, 09/05/1985). En esta declaración, a partir de las repreguntas, Eposto agrega más información a su testimonio. Ser militar le había posibilitado ingresar con mayor libertad al servicio de epidemiología. En su declaración aparece el siguiente relato: “[a las embarazadas] las pudo observar (...) en horas de la tarde (...) y se solía trasladar por su propia voluntad hasta las dependencias del Servicio de Epidemiología para enterarse de lo que pasaba en dicho lugar sólo a los efectos de satisfacer su curiosidad; que en tales circunstancias, accedía al interior del pasillo al que daban las puertas de las habitaciones de las internadas con la excusa y el pretexto de ir a conversar con el suboficial que se encontraba allí normalmente apostado como custodia; que al suboficial al que hace referencia era un suboficial del Ejército y no de Gendarmería al que, por pertenecer al personal destinado al Hospital Militar, el dicente siempre conocía (...)”. (Eposto, JM, 09/05/1985). La apelación a la curiosidad que introduce Eposto resulta llamativa. Más allá de la caracterización moral que se quiera hacer, abre una serie de interrogantes: ¿eran franqueables las puertas del servicio de epidemilogía de forma sencilla? ¿aún en la excepcionalidad reinante, los lazos de camaradería tuvieron un peso tan alto como para permitir a alguien ingresar a un lugar prohibido? La imagen que se puede construir a partir del relato es muy diferente de la idea de organización y marcialidad que suele dominar el imaginario sobre la dictadura.

Después de la primera declaración frente a la justicia militar, en octubre, Eposto vuelve a ser convocado a declarar con el objetivo de precisar los datos que ha brindado. La declaración no varía mucho ya que Eposto evade las respuestas de forma sistemática. Sin embargo, cabe destacar algunos pasajes del testimonio que dan cuenta de un momento transicional en el que aún se están consolidando algunos sentidos sobre el pasado. En su declaración frente a CONADEP, Eposto hace referencia a la existencia de Grupos de Tareas en los que participaba un enfermero "para brindar asistencia al personal del Grupo de Tareas" y, según indica, lo sabe por "entre otras referencias por un enfermero que iba al Hospital y contaba su participación en el Grupo de Tareas" (Eposto, CONADEP, 30/01/1984). Si bien no es interrogado sobre esta cuestión en la primera declaración, en esta oportunidad se le pregunta "de qué delito cree poder acusar (...) [a] los llamados grupos de tarea". Eposto, señala que "no le consta que (...) durante sus actuaciones en la lucha contra la subversión haya cometido algún tipo de delito, desmán o exceso" (Eposto, JM, 24/10/1985). Si bien es evidente que para 1985 ya era claro el accionar de los Grupos de Tarea, e incluso es posible inferir que Eposto conoce el rol que éstos cumplieron en el sistema represivo y por eso lo menciona en CONADEP, resulta significativo que aún haya margen para interrogar sobre esta cuestión; más aún cuando en CONADEP no se repreguntó al respecto y tampoco en el primer interrogatorio en la justicia militar.

Otro pasaje destacable de la declaración, es cuando Eposto, al final, de forma espontánea, hace algunas aclaraciones sobre su declaración en CONADEP. En primer lugar indica que se negó a que apareciera su nombre pero que igualmente 


\section{Clara Sarsale}

fue incluido "en el libro "Nunca más", (...) en uno de los párrafos de más notoriedad, haciendo evidentemente caso omiso a su particular deseo (...) de que el nombre no fuera insertado en la publicación" (Eposto, JM, 24/10/1985). Además, señala Eposto, que el Dr. Aragón, secretario de CONADEP, le habían dicho que poner su nombre le podría traer beneficios. En segundo lugar, aclara que "el motivo de la «presentación expontánea» (sic) [que hizo frente a CONADEP] tuvo su origen en el deseo de obtener el cumplimiento de una promesa que le hiciera el señor Pimentel, en el sentido de que si efectuaba una denuncia importante, se trataría de favorecer al dicente con un puesto Nacional" (Eposto, JM, 24/10/1985). Los dos pasajes citados no habían sido expuestos frente a la justicia civil ni en la primera declaración ante el tribunal militar por lo que merece considerarse el motivo por el que, en esta oportunidad, Eposto hace pública esta información. Declarar frente a la justicia militar es, en cierto grado, declarar ante pares, en la propia corporación. Los beneficios que le habrían ofrecido, el desconocimiento sobre la ilegalidad de los grupos de tareas, pueden leerse como una manera de excusar su comportamiento fuera del círculo militar, de lo que puede ser leído como una traición. En este sentido, más allá de la veracidad o no de los hechos declarados, los cambios en las declaraciones se van resignificando en función del interlocutor y el espacio en que éstas se producen.

Mientras que en CONADEP los dichos de los testimoniantes no eran cuestionados, al menos no de forma inquisitiva, el tribunal militar fue un espacio hostil para quienes debían enfrentarlo. Esto fue así para Eposto, pero también para Nélida Valaris, una obstétrica civil que trabajaba en el Hospital de Campo de Mayo. Valaris, se presentó a declarar en CONADEP en abril de 1984. Valaris tuvo que atender dos partos clandestinos durante la dictadura. El primero de ellos fue en la Cárcel de encausados que funcionaba al interior del enorme predio de Campo de Mayo $^{12}$. En su testimonio frente a CONADEP (que luego formará parte del texto del "Nunca más") Valaris contó que el parto se produjo en agosto de 1977 en la enfermería de la cárcel, a la que fue llevada en una camioneta. La sala donde se hallaba la mujer rubia y joven, estuvo siempre llena de hombre de fajina y de civil. También contó, que estos recuerdos le quedaron grabados "porque la situación la desbordó" (Valaris, CONADEP, 1984) y que "se negó rotundamente a concurrir [a la Cárcel de encausados] recibiendo la orden directa de Di Benedetto, director del Hospital" (Valaris, CONADEP, 1984).

El relato de Valaris en términos de experiencia dista del de Eposto; frente a la apelación de la curiosidad en el caso de Eposto, aparece la negativa de la obstétrica a atender el parto, incluso exponiéndose a discutir con su superior, el Dr. Caserotto. Las diferencias se evidencian en las respuestas que brinda cada uno frente a la Justicia Militar. La declaración de Valaris es extensa; se le pregunta con gran detalle por el estado de situación de las embarazadas, por el funcionamiento del Hospital, los traslados de las embarazadas, entre otras preguntas. La primera

12 En la declaración que Valaris realiza en 1998, brinda muchos más datos y precisiones sobre este parto. 


\section{Las primeras declaraciones de los profesionales de la salud del Hospital de Campo de Mayo}

de ellas apunta desentrañar la negativa con que Valaris respondió frente a uno de los partos; el tribunal le pidió que "manifieste con el mayor detalle las causas por las cuales se negara inicialmente a concurrir a la prisión militar de encausados, orden dada por el Doctor Caserotto para asistir a una mujer detenida que se encontraba en situación de dar a luz" (JM 21/05/1985). Según testimonia Valaris, su negativa se vincula a "que la actividad que se le demandaba no era la que le correspondía en razón de los servicios para los que se halla contratada. (...) que en la circunstancia temió que por tener que trasladarse a cumplir la orden que se le daba (...) pudiera darse alguna urgencia en su servicio que no pudiera atender por encontrarse alejada del mismo. Que además la asaltó el temor de tener que enfrentar alguna urgencia que escapara a las posibilidades de su idoneidad (...); que asimismo consideró que por tratarse de la clase de paciente que se tenía que asistir, le hubiera tocado cumplir la tarea al Dr. Caserotto o al Dr. Lederer" (Valaris, JM, 21/05/1985). Como en el caso de Eposto, frente al tribunal militar, Valaris también se ve obligada a explicar su accionar. Lo que en la CONADEP no era cuestionable ya que se infería que Valaris se opuso en tanto se trataba de atender un parto ilegal, si lo era para la Justicia Militar. Valaris recurrió a argumentos puramente profesionales o burocráticos; como señalé en otra oportunidad (Sarsale, 2014), la resistencia se estructura a partir de la obediencia a la norma y no de la ruptura de ésta. Cabe agregar para analizar la respuesta de Valaris, que ella seguía trabajando en el Hospital y que había sido amenazada antes de ir a declarar a CONADEP por quienes seguían siendo sus superiores, el Dr. Caserotto y el Dr. Di Benedetto ${ }^{13}$. La respuesta que dió, no fue sólo una explicación para su accionar pasado, sino en gran medida, una protección para su presente, resguardándose en la obediencia que caracteriza a la disciplina militar.

Nélida Valaris no fue la única que se opuso a atender un parto; la Dra. Bonsignore de Petrillo es otra de las profesionales que lo hizo según declaró de forma espontánea en CONADEP el mismo día que Valaris, el 10 de abril de 1984. La Dra. Bonsignore atendió dos partos de "parturientas que estaban vendadas, que no se identificaban" (Bonsignore, CONADEP, 1984). Uno de los partos lo atendió junto a Valaris; el otro caso que tuvo que atender" fue una cesárea. La Dra. Bonsignore se encontraba realizando guardia pasiva en su hogar cuando recibe el llamado de emergencia para ir a atender una cesárea. Al llegar a la sala donde debía cambiarse para ingresar al quirófano se encuentra, para su sorpresa, con personal vestido de fajina entre quienes se encontraba el Dr. Bianco ${ }^{14}$. En la declaración que brindó

\footnotetext{
13 Las amenazas no fueron denunciadas por Valaris ni frente a CONADEP, ni en sus primeras declaraciones, a diferencia de Eposto que lo denuncia rápidamente frente a la justicia civil. Recién en 1998, Valaris empieza a hacer referencia a las mismas; para esa fecha ya no trabajaba en Campo de Mayo.

14 El Dr. Bianco de especialidad traumatólogo cumplió un rol fundamental en la estructuración de la maternidad clandestina. En múltiples declaraciones se lo señala llevando y trayendo del sector de epidemiología a las embarazadas. Además, según refiere Bonsignore, durante los años 76 y 77 no ejerció como médico pasando a usar ropa de fajina y dedicarse al traslado de heridos de todo tipo y
} 


\section{Clara Sarsale}

ante la Justicia Militar, Bonsignore aclaró que quien le dio la orden para practicar la cesárea fue el Dr. Caserotto, jefe del servicio sin embargo ella se negó. Es llamativo que no sea interrogada sobre las razones de esta negativa. Tal vez, la explicación yace en el relato que han hecho posteriormente colegas de Petrillo. En el juicio del 2011, el Dr. Poisson ha declarado que "la Dra. Petrillo fue obligada por Caserotto a hacer una cesárea en una paciente que no pudo identificar, que no tenía historia clínica, que no debió inscribir en los libros. (...) Ella fue obligada a punta de pistola. (...)" (Poisson, Plan Sistemático, 2011). En este mismo sentido, otro médico, Raffinetti también en 2011 contó que "lo que sé es que la obligaron, prácticamente la levantaron de los pelos para atender a esa persona" (Raffinetti, Plan Sistémico, 2011) ${ }^{15}$. Teniendo en cuenta estas declaraciones es posible pensar que el tribunal militar preguntaba más en la medida en que las respuestas no fueran perjudicial para la corporación. Por un lado, el tribunal militar no preguntó y por el otro, tampoco Bonsignore mencionó las amenazas. Es lógico pensar que, aunque ya se había instalado la democracia, las fuerzas armadas seguían conservando un enorme poder simbólico y real. Y este factor debe ser considerado para los tres testimonios donde las amenazas se hacen presentes de una u otra manera. Sin embargo, y en línea con lo que sucedió durante el período dictatorial, las amenazas y el temor no explican todo. Como se ha visto, aún en ese contexto represivo, los sujetos rechazan, se oponen, sienten curiosidad, circulan información.

\section{Síntesis y nuevas preguntas.}

¿Qué permiten pensar estos pequeños fragmentos de testimonio? En primer lugar, dan cuenta de la coyuntura histórico política en la que se inscriben que, como dije anteriormente, está signada por una disputa entre el poder civil y el de las FF.AA. expresado en este caso en la disputa entre la Justicia civil y militar. Esta disputa se traduce en las múltiples declaraciones que tienen que hacer los testimoniantes ratificando una y otra vez las declaraciones precedentes. En segundo lugar, es notorio que los testimoniantes tienen en cuenta quiénes son sus interlocutores y establecen estrategias a partir de esa información. En este sentido, el poder que aún retenían las Fuerzas Armadas, y en especial en el caso de quienes todavía trabajaban en el Hospital impacta en los testimonios. La denuncia de haber sido amenazado que hace Eposto y la amenaza que hoy se sabe recibieron Valaris y Bonsignore son indicios del ejercicio de ese poder amedrentador que tenían las FF.AA.

En tercer lugar, y en línea con el interés de este texto, los testimonios se presentan como una puerta para pensar sobre los modos de actuar durante la dictadura. En

embarazadas (Bonsignore, JC - San isidro 21/08/1984). En 2014, Bianco recibió la última condena por apropiación de menores junto con la obstetra Arroche, Riveros y Bignone.

${ }^{15}$ Aclaración: no he podido acceder aún a la declaración de Bonsignore en el Juicio realizado en 2011. 


\section{Las primeras declaraciones de los profesionales de la salud del Hospital de Campo de Mayo}

los testimonios analizados se encuentra, por un lado, la referencia a la curiosidad por parte de Eposto respecto de lo que estaba sucediendo. Por el otro, dos de las testimoniantes se han opuesto al menos en una primera instancia a atender los partos tal y cómo se los demandaban sus superiores. Esta situación, en la década del ochenta, probablemente encontraba escasa repercusión enmarcada en el gran relato que proveyó la Teoría de los dos demonios que inocentizaba a la sociedad. En este sentido, no se trataba de familiares de víctimas cuyo enfrentamiento a las autoridades podía explicarse en relación a su historia familiar, ni tampoco de una resistencia llevada adelante por militantes, o en los últimos años de la dictadura cuando ésta se encontraba debilitada. Lo que se está señalando es la existencia de algún grado de oposición a las órdenes militares en plena guarnición militar y la paradoja de que estas acciones de oposición, contra lo imaginable, no conllevaron reprimendas por parte de los militares ni siquiera en términos de sumarios administrativos ${ }^{16}$. Retomando las perspectivas historiográficas con las que se dio inicio a este trabajo, podemos ver a estos individuos como sujetos activos, coproductores de las situaciones en las que participan y no meros ejecutores de las órdenes ajenas. Las dos mujeres que se niegan a atender partos, no logran su cometido, el poder de las autoridades se impone sobre ellas. Sin embargo, contra la interpretación de la sociedad como víctima que aparece en el Nunca más, o como aquellas que la describen como cómplice, estos casos muestran otros márgenes de acción. El caso de Eposto es también elocuente en este sentido. Este radiólogo no es obligado a ir y mirar lo que pasa al interior de epidemiología, ni se requieren sus servicios, ni se demanda que hable con el personal que custodia el lugar. Pero él lo hace de todas formas motivado por su propia curiosidad, por su propio interés. Es esa su manera de apropiarse de una situación claramente excepcional.

El análisis aquí propuesto no permite establecer ni una conclusión inamovible, ni generalizable. Sin embargo, permite abrir la trama del tejido, ver los hilos que la componen, sus interrelaciones y diferencias. La ampliación de este análisis a más testimonios $\mathrm{y}$ fuentes permitirá continuar con esta problematización.

\section{Bibliografía}

Alejandro Baer. Holocausto. Recuerdo y representación. Madrid, Ed. Losada, 2006. Cristopher Browning. Aquellos hombres grises. El batallón 101 y la solución final en Polonia. Buenos Aires, Ed. Edhasa, 2011.

Alf Lüdtke. "De los héroes de la Resistencia a los coautores. 'Altagsgechichte'Alemania" en Luis Castells (ed.) La historia de la vida cotidiana, Madrid Marcial Pons, 1995.

\footnotetext{
${ }^{16}$ Me refiero exclusivamente al caso del Hospital de Campo de Mayo, ya que - como es sabido - en otras dependencias como el Hospital de Quilmes, Generosa Fratassi, una enfermera del Hospital, fue desaparecida por informar el nacimiento de una niña cuya madre se encontraba desaparecia.
} 


\section{Clara Sarsale}

Ana Inés Seitz; Valeria Durán; Federico Iglesias; Sabrina Ríos; Laura Schenquer, "Decir no sólo miedo y represión quiere decir que también el miedo y la represión formaron parte del franquismo". Páginas. Revista digital de la Escuela de historia UNR, N.o 10, Año VI, Rosario, 2014, pp. 151-161. Disponible en http://rephip.unr.edu.ar/bitstream/handle/2133/4725/337-13161-PB.pdf?sequence=1

Clara Sarsale. "Testigos involuntarios entre víctimas y victimarios. Reflexiones en torno a tres testimonios del Juicio por la Verdad en Mar del Plata". Prácticas de oficio. Investigación y reflexión en Ciencias Sociales. №13, 2014. ISSN 1851-5636. Disponible en: http://ides.org.ar/publicaciones/practicasdeoficio/practicas-deoficio-reflexion-e-investigacion-en-ciencias-sociales-nro-13

Claudio Hernández Burgos. Las bases sociales de la dictadura y las actitudes ciudadanas durante el régimen de Franco, Granada (1936 -1976). Granada, Ed. Universidad de Granada, 2012.

CONADEP (1984), Nunca más. Informe de la Comisión Nacional sobre la desaparición de personas. Ed. Eudeba Buenos Aires.

Daniel Goldhagen. Los verdugos voluntarios de Hitler. Los alemanes corrientes y el Holocausto. Madrid, Ed. Taurus, 1998.

Daniel Lvovich. "Actitudes sociales y dictaduras: las historiografías española y argentina en perspectiva comparada". Páginas. Revista digital de la Escuela de historia - UNR, N. I, Año I Rosario, 2008, pp.21 - 49. Disponible en http://revistapaginas.unr.edu.ar/index.php/RevPaginas/article/view/149

Emilio Crenzel. "Dos prólogos para un mismo informe: el Nunca más y la memoria de las desapariciones”. Prohistoria №.11. Rosario, 2007, pp.49 -60. Disponible en:

Federico Finchelstein. Los alemanes, el holocausto y la culpa colectiva. El Debate Goldhagen. Buenos Aires, Ed. Eudeba, 1999.

Ian Kershaw. La dictadura nazi. Problemas y perspectivas de investigación., Buenos Aires, Ed. Siglo XXI, 2004.

Ismael Saz Campos. Fascismo y Franquismo. Valencia, Ed. Universitat de Valencia, 2004.

\section{Referencia fuentes}

JM: Justicia militar

Plan sistemático: Juicio llevado adelante en el Tribunal Oral Federal $\mathrm{n}$-6 durante el 2011 y el 2012 en el que se juzgó a algunos militares entre los que se encontraban Videla y Bignone por la aplicación de un Plan sistemático de apropiación de menores durante la última Dictadura.

CONADEP. Comisión Nacional sobre la desaparición de personas.

Recibido: 20/06/2016

Evaluado: $17 / 07 / 2016$

Versión Final: 24/08/2016 